\title{
Polyhydramnios in COVID-19 infected Pregnant Woman
}

\author{
Yeshey Dorjey ${ }^{1}$ and Deep Chhettri² \\ ${ }^{1}$ Royal Government of Bhutan Ministry of Health Department of Public Health \\ ${ }^{2}$ Royal Government of Bhutan Ministry of Health
}

October 27, 2021

\begin{abstract}
Bhutan has a total of 2006 cases (1271 Male, 735 Females) with one death[3]. Among 735 females, four pregnant mothers had COVID-19 infection. One mother infected with COVID-19 at 16 weeks of gestation developed polyhydramnios. The present study reports a rare finding of polyhydramnios in COVID-19 infected pregnant woman.
\end{abstract}

\section{Polyhydramnios in COVID-19 infected Pregnant Woman}

Types of the article: A case report

Yeshey Dorjey $^{{ }^{*}}$, Deep Kiran Chhetri ${ }^{2}$

1.Consultant Obstetricians \& Gynaecologist, Gynecology unit, Phuentsholing Hospital, Bhutan

2.Consultant Obstetricians \& Gynecologist, Gynecology unit, Tashigang Hosptial, Bhutan

*Corresponding author:

Yeshey Dorjey, Phuentsholing Hospital, Post office: Phuentsholing, Chukha, Bhutan.

E-mail address: yesheydorjey@gmail.com

https://orcid.org/0000-0001-7324-3028

\section{Synopsis}

Polyhydramnios in COVID-19 infected pregnant mother is not reported in the literature. The placental insult caused by the COVID-19 infection could the causing polyhydramnios.

\section{Keyword}

Polyhydramnios; COVID-19 infection; pregnant mother

\section{INTRODUCTION}

By December 2019, the first case of COVID-19 was reported in Wuhan city of China[1]. COVID-19 spreads rapidly affecting all including pregnant mothers. The first case of COVID-19 was detected in Bhutan on $5^{\text {th }}$ March 2020[2]. Bhutan has a total of 2006 cases (1271 Male, 735 Females) with one death[3]. Among 735 females, four pregnant mothers had COVID-19 infection. One mother infected with COVID-19 at 16 weeks of gestation developed polyhydramnios in pregnancy. The present study reports a rare finding of polyhydramnios in COVID-19 infected pregnant mothers.

\section{Case presentation}


A 29-year-old primigravida at 16 weeks of gestation was diagnosed with a COVID-19 infection. Her dating scan was done at $10^{+4}$ weeks gestation. She did not have medical or surgical co-morbidities.

At 16 weeks of gestation on $17^{\text {th }}$ August 2020, she was tested for COVID-19 as one of the primary contacts of Mini Dry Port (MDP) loaders who tested positive. Her reverse transcriptase-polymerase chain reaction (RT-PCR) test was confirmed positive. She was admitted to Royal Institute for Governance and Strategic Studies (RIGSS) isolation hospital on $18^{\text {th }}$ August 2020. She was asymptomatic on admission; her vital parameters were unremarkable. Her chest x-ray showed the normal study. Vitamin supplementations were continued during her pregnancy.

She was followed up daily for signs and symptoms of COVID-19 and obstetrical complications. Repeat RT-PCR was done as per the testing protocol of the country. On day-7 of isolation, she developed a mild fever with shortness of breath and a loss of smell. Vital signs got slightly deranged; Temperature 40-degree Celsius, respiratory rate 28 breaths/min, $\mathrm{SpO} 2$ dropped to $94 \%$, pulse rate 96 beats/min, and BP slightly raised (Figure 1). Oxygen was administered by face mask at 6-8 L/min and conservatively managed. After 4-6 hours of oxygenation, all the vital signs were restored to normal and her symptom of shortness of breath subsided. From day-8 onward the patient was clinically stable and biochemically normal.

On day-35 RT-PCR test for COVID-19 was negative and confirmed on day-36 with a repeat RT-PCR. The patient was shifted to the de-isolation facility on day-37 and kept for two weeks. The repeat RT-PCR was performed at the end of de-isolation and sent for home quarantine for another week and kept under COVID-19 surveillance by the ministry of health, Bhutan.

From 24 weeks of gestation, she was followed up at the Maternal and Child Health (MCH) care unit, Phuentsholing hospital. She had made six antenatal visits. Her pregnancy was uneventful until 36 weeks of gestation where she was diagnosed to have polyhydramnios with an Amniotic Fluid Index (AFI) of $29 \mathrm{~cm}$ (Figure 2).

The patient was evaluated for the cause of polyhydramnios: Oral glucose tolerance test with $75 \mathrm{~g}$ glucose done, FBS $5.0 \mathrm{mmol} / \mathrm{L}$ and 2 hours after glucose $7.5 \mathrm{mmol} / \mathrm{L}$; Viral markers all negative; detailed anatomical scan showed no significant fetal anomalies seen. Pregnancy was terminated at 39 weeks of gestation by cesarean section for failed induction. A live healthy female baby weighing $3550 \mathrm{~g}$ was delivered with the Apgar score of 8 in 1 minute and 10 in 5 minutes. At birth no gross anomaly or birth defects were present. After 48-hours of delivery, an ultrasound scan of the newborn was done and ruled out gastrointestinal tract anomalies. The baby was feeding well and perfectly normal.

\section{Discussion}

In Bhutan, the rate of COVID-19 infection in pregnancy is low as compared to $27.8 \%$ in India[4]. The low rate of infection during pregnancy in Bhutan is due to stringent control measures in place to curb the spread of COVID-19 infections.

The majority of the pregnant mothers got infected with COVID-19 in their second and third trimesters [5], which is comparable to the current case.

Preterm delivery is the most reported perinatal effect of COVID-19 infection during pregnancy[6]. Polyhydramnios in COVID-19 infection is not reported in the literature. In the current case, a pregnant mother got infected with COVID-19 at 16 weeks of gestation and developed polyhydramnios later in the pregnancy. The exact cause of polyhydramnios could not be established. Viral infection during pregnancy causes the release of cytokines and cause oxidative stress and disrupts the placental functions and causes polyhydramnios[7]. Since COVID-19 is a viral infection, it may have caused placental insult due to cytokine storm during the pregnancy and resulted in polyhydramnios. The detailed effects of COVID-19 at biochemical levels in the body were studied. However, the effects of COVID-19 at the placenta are not studied; the exact effects of the COVID-19 virus on the placenta remain uncertain because it is a new disease and detailed information is not available in the literature. 
This case concludes polyhydramnios may have been caused by COVID-19 infection during pregnancy due to placental insult and oxidative stress.

\section{Acknowledgments}

The author is grateful to Dr. Tshewang Gyeltshen (Dental surgeon, Tsirang District Hospital, Bhutan) for reviewing the manuscript and providing feedback.

\section{Author Contributions}

YD performed the clinical diagnosis, conceived the idea, drafted the manuscript, and submitted the final version of the manuscript for publication.

DPC conceived the idea, collected the clinical data, revised the manuscript, and submitted it for publication.

\section{ORCID}

Yeshey Dorjey https://orcid.org/0000-0001-7324-3028

\section{Conflicts of Interest}

The authors have no conflicts of interest.

\section{Informed consent}

Informed consent was obtained from the patient for the collection of case history and publication of this case report along with ultrasound images. The patient consent has been signed and collected in accordance with the journal's patient consent policy.

\section{References}

[1] WHO, "Coronavirus disease 2019 (COVID-19) Situation Report - 94," 2020. [Online]. Available: https://apps.who.int/iris/handle/10665/331865.

[2] S. LeVine et al. , "Case report: The first case of COVID-19 in Bhutan," Am. J. Trop. Med. Hyg. , vol. 102, no. 6, pp. 1205-1207, 2020, doi: 10.4269/ajtmh.20-0259.

[3] MOH, "National Situational Update on COVID-19," Ministry of Health, Bhutan , 2021. http://www.moh.gov.bt/national-situational-update-on-covid-19-95/\# (accessed Jun. 25, 2021).

[4] I. News, "Hindustan Time," India News, New Delhi, Jun. 17, 2021.

[5] A. Khalil et al. , "SARS-CoV-2 infection in pregnancy: A systematic review and meta-analysis of clinical features and pregnancy outcomes," EClinicalMedicine, vol. 25, no. December 2019, p. 100446, 2020, doi: 10.1016/j.eclinm.2020.100446.

[6] K. R. Woodworth et al. , "Birth and Infant Outcomes Following Laboratory-Confirmed SARS-CoV-2 Infection in Pregnancy - SET-NET, 16 Jurisdictions, March 29-October 14, 2020," MMWR. Morb. Mortal. Wkly. Rep., vol. 69, no. 44, pp. 1635-1640, 2020, doi: 10.15585/mmwr.mm6944e2.

[7] K. M. Adams Waldorf and R. M. McAdams, "Influence of infection during pregnancy on fetal development," Reproduction, vol. 146, no. 5, 2013, doi: 10.1530/REP-13-0232.

\section{Hosted file}

Figure 1.docx available at https://authorea.com/users/419807/articles/543288-polyhydramniosin-covid-19-infected-pregnant-woman

\section{Hosted file}


Figure 2.docx available at https://authorea.com/users/419807/articles/543288-polyhydramniosin-covid-19-infected-pregnant-woman 